stabilized (Fig. 1d). As $\mathrm{SpO}_{2}$ remained at 80-89\%, extubation was performed at 16 days after the event. On the next day, she was transferred from the ICU. She was discharged home because circulation and respiration were stable, with no sequelae or relapse of epileptic seizure. We received written consent from the child's parents when we submitted this paper.

There are no reported cases in which ACTH therapy leads to outflow tract obstruction with a ventricular conduit in patients undergoing the Norwood procedure with the RV-PA conduit, as in our case. Adrenocorticotrophic hormone therapyinduced right ventricular myocardial hypertrophy possibly resulted in ostial stenosis of the right ventricular side of the RV-PA conduit, leading to a decrease in pulmonary blood flow and circulatory failure. With ECMO support and conduit clip removal using a balloon dilatation catheter, she survived the myocardial hypertrophy exacerbation. Myocardial hypertrophy with ACTH therapy is usually reversible. We therefore chose to remove the clips of the conduit with a balloon catheter, rather than using stent implantation between the RV-PA conduit and hypertrophic myocardium. Adrenocorticotrophic hormone therapy can lead to complications, including myocardial hypertrophy. Bobele et al. reported that $73 \%$ of patients receiving ACTH therapy had reversible myocardial hypertrophy. ${ }^{1} \mathrm{We}$ thought that administration of a beta-blocker might reduce hypertrophy. However, she had unstable vital signs and her $\mathrm{SpO}_{2}$ level was variable and depended on her blood pressure; thus, we could not use a beta-blocker. In patients undergoing the Norwood procedure with the RV-PA conduit, stenosis of the right ventricular outflow tract has poor prognosis, leading to decrease in pulmonary blood flow and fatal circulatory failure, requiring catheter intervention or surgery. ${ }^{4,5}$ Early detection of myocardial hypertrophy with frequent monitoring is clearly important, termination or reduction of ACTH therapy depending on the severity of myocardial hypertrophy should also be considered. Myocardial hypertrophy is temporary, so circulatory control with ECMO support is useful for circulatory failure. For these reasons, when ACTH therapy is performed for children after the Norwood + RV-PA conduit operation, this should be done in facilities that can be treated intensively.

\section{Disclosure}

The authors declare no conflicts of interest.

\section{Author contribution}

M.K. wrote the manuscript. D.H. gave conceptual advice and supervised the whole study process. S.M., K.U., and Y.K. critically reviewed the manuscript. All authors read and approved the final manuscript.

\section{References}

1 Bobele GB, Ward KE, Bodensteiner JB. Hypertrophic cardiomyopathy during corticotropin therapy for infantile spasms. A clinical and echocardiographic study. Am. J. Dis. Child. 1993; 147: 223-5.

2 Hishitani T, Hoshino K, Ogawa K et al. Rapid enlargement of cardiac rhabdomyoma during corticotropin therapy for infantile spasms. Pediatr. Cardiol. 1997; 13: 72-4.

3 Young RS, Fripp RR, Stern DR et al. Cardiac hypertrophy associated with ACTH therapy for childhood seizure disorder. J. Child Neurol. 1987; 2: 311-2.

4 Nigro JJ, Bart RD, Derby CD et al. Proximal conduit obstruction after Sano modified Norwood procedure. Ann. Thorac. Surg. 2005; 80: 1924-8.

5 Benjamin P, Tory A, Thomas K, Gordon C, Christer J. Case report - Resistant hypoxemia in an infant with a right ventricleto-pulmonary artery (Sano) shunt. J. Cardiothorac Vasc Anesth. 2007; 21: 880-2.

\title{
Mycobacterium tuberculosis prosthesis joint infection
}

\author{
Andreia Fiúza Ribeiro, ${ }^{1}$ (D) Marisa Inacio Oliveira, ${ }^{1}$ Pedro Jordão, ${ }^{2}$ Delfin Tavares,${ }^{2}$ Luís Varandas ${ }^{1}$ and \\ Catarina Gouveia ${ }^{1}$ \\ ${ }^{1}$ Infectious Diseases Unit and ${ }^{2}$ Pediatric Orthopedic Unit, Área da Mulher, Criança e Adolescente, Área da Mulher, \\ Criança e Adolescente, Hospital Dona Estefânia, Centro Hospitalar Universitário Lisboa Central, EPE, Lisbon, Portugal
}

Key words chronic osteomyelitis, hip arthroplasty, prosthesis infection, tuberculosis.

Correspondence: Catarina Gouveia, MD, Infectious Diseases Unit, Área da Mulher, Criança e Adolescente, Hospital de Dona Estefânia, Centro Hospitalar Universitário Lisboa Central - EPE, Lisbon, Portugal, Rua Jacinta Marto, 1169-045 Lisboa. Email: cmfgouveia@gmail.com

Received 17 June 2018; revised 10 July 2019; accepted 3 September 2019.

doi: $10.1111 /$ ped.14037
Prosthetic joint infection (PJI) is an uncommon, dreadful complication of hip arthroplasties. Mycobacterium tuberculosis (TB) is a rare causative agent. ${ }^{1}$

We present a 17-year-old male teenager from Angola, living in Portugal for 3 years, admitted with hip PJI. At 5 years old right hip instability was noted after septic arthritis in Angola. In July 2015, at 16 years old, he underwent total right-hip 


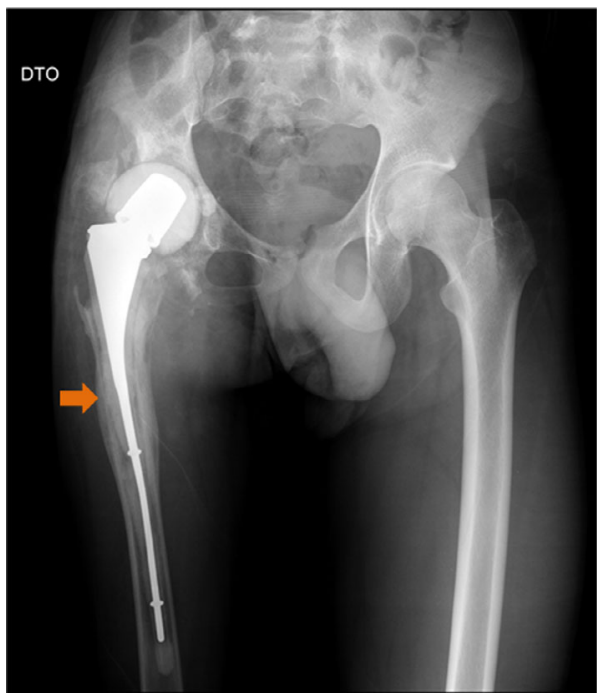

Fig. 1 Hip X-Ray before spacer removal. The X-Ray demonstrates extensive metadiaphyseal bone loss, with substantial diaphysis osteolysis (arrow) of the right femur, suggestive of chronic osteomyelitis. Acetabular roof with multiple bone fragments.

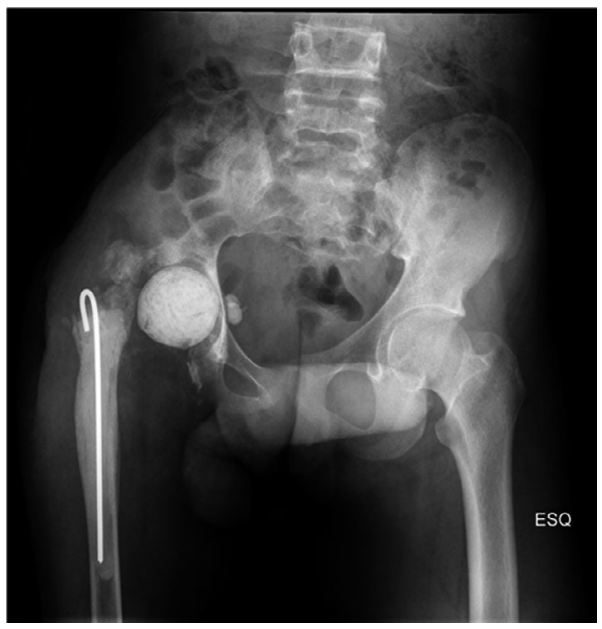

Fig. 2 Hip X-Ray after spacer removal. The X-Ray demonstrates extensive metadiaphyseal bone loss, with substantial diaphysis osteolysis of the right femur, suggestive of chronic osteomyelitis. Acetabular roof with multiple bone fragments.

arthroplasty in another Portuguese hospital, due to femoral head necrosis. Two months later PJI was diagnosed, positive for Staphylococcus aureus (in three different samples), Acinetobacter baumannii, and Pseudomonas aeruginosa (both in one sample). He was sequentially treated with antibiotics, debridement with retention and in September 2016 implant removal and placement of a spacer. He was admitted to our hospital in November 2016, with 1 week evolution of fever, hip pain, and two fistulas at the right buttock. He had been being treated with flucloxacillin (500 mg tid) for the previous 2 months, with irregular compliance. He denied weight loss, night sweats, anorexia, and respiratory symptoms. A sequelar $10 \mathrm{~cm}$ lower limb dysmetria and a foot drop were noted.

Laboratory studies showed leukocytosis (15 120/L; Reference value (RV): 4,500-11 000/L), a high C-reactive protein (147 mg/L; RV: $<5 \mathrm{mg} / \mathrm{L}$ ) and a positive interferon-gamma release assay (IGRA) (QuantiFERON - TB-gold). The hip Xray (Figs 1,2) and pelvic computed tomography revealed chronic osteomyelitis with multiple peri-prosthetic abscesses fistulized to the buttock. He was started on piperacillin / tazobactam and vancomycin and underwent surgical debridement, spacer removal, and placement of gentamycin-impregnated cement.

Bone histopathology revealed unspecific inflammation; acidfast staining and mycobacterial nucleic acid amplification test were negative, and culture was positive for extended spectrum beta lactamase (ESBL)-producing Klebsiella pneumonia. Therapy was changed to meropenem (1 g tid) but at D37 bone culture turned positive for Corynebacterium jeikeium (on Lowenstein, no susceptibility test) and Mycobacterium tuberculosis, resistant to isoniazid and streptomycin. Pulmonary TB and HIV infection were excluded and he was started on rifampicin (600 mg id), pyrazinamide (1,500 mg id), levofloxacin $(750 \mathrm{mg}$ id), and ethambutol (900 mg id). At 18 months he stopped treatment and currently has no pain or fistula and is waiting for a second-stage arthroplasty. Treatment was well tolerated, with minor hepatic toxicity (ALT 2-3 times higher at 12 months).

In Portugal, TB incidence was 15.6/100 000 inhabitants in 2017, being much higher in immigrants (four to eight times higher $)^{2}$ and even higher in Angola (359/100 000 inhabitants in 2017). ${ }^{3}$ Although infrequent, 83 cases of TB PJI were reported, usually involving the hip or knee, but none in childhood. ${ }^{1}$ A prior TB or immigration history, immunomodulatory therapy or failing to respond to conventional treatment should prompt for TB. Although typical, the characteristically caseating granulomas explain diagnosis in only $4.5 \%$ of patients. ${ }^{4}$ Microbiological exams are the mainstay for diagnosis; however, sensitivity is lower in children $(61-74 \%) .^{5}$ In our case TB might have been acquired by hematogenous spread of a latent TB, as only $30 \%$ of patients have past TB history, ${ }^{4}$ or by local reactivation during surgery.

Treatment options are controversial. Veloci et $a l^{4}$ reported that initial stage TB PJI, as in our case, usually responds well to standard TB regimens, with no need for extensive surgery. Indeed, Mycobacterium tuberculosis lower adherence and scarce biofilm formation over prosthetic material might explain this favorable response. ${ }^{4}$ In late-onset infections, surgical reconstruction is often required due to substantial bone loss and disease progression. In our case, the extensive bone loss and concomitant bacterial PJI added to the isoniazid resistance further compromised the outcome, justifying a more aggressive approach. In fact, Veloci et al. reviewed 66 patients with TB-PJI, $80.3 \%$ submitted to surgical treatment. Surgical options varied from debridement (17\%), to exchange $(7.6 \%)$, two-staged exchange $(22.7 \%)$ or prosthesis removal $(33 \%)$. However, in most patients, prosthesis revision was done before 
TB diagnosis, suggesting that a more conservative approach (medical therapy with or without debridement) should be tried first in these patients. ${ }^{4}$

The role of co-pathogens is unknown, although exclusive TB treatment seems successful in most cases. ${ }^{4}$ However, we chose to treat Klebsiella ESBL strain with meropenem for 4 weeks. Coryneform bacilli are usually considered as contaminants in clinical samples, however they has been implicated in PJI, mostly C. jeikeium. In our case, although antimicrobial susceptibility was not possible, the patient was initially treated with vancomycin and later with levofloxacin and rifampicin, which cover most $C$. jeikeium strains.

TB-PJI is uncommon and the length of medical treatment and the need for prosthesis removal is uncertain, although a more conservative approach could be chosen in the initial stages.

\section{Disclosure}

The authors declare no conflict of interest.

\section{Author contribution}

A.F.R. and C.G. wrote the manuscript; C.G., M.I.O., P.J., D.T., and L.V. critically reviewed the manuscript.

\section{References}

1 Uhel F, Corvaisier G, Poinsignon Y et al. Mycobacterium tuberculosis prosthetic joint infections: a case series and literature review. J Infect. 2019; 78: 27-34.

2 Tuberculose em Portugal - Desafios e Estratégias 2018. Direção-Geral da Saúde, Março, 2018. [Cited 2018 Nov 8]. Available from: https://www.dgs.pt/paginas-de-sistema/saudede-a-a-z/tuberculose1/relatorios.aspx

3 WHO Tuberculosis country profiles. [Cited 2018 Nov 8]. Available from: http://www.who.int/tb/country/data/profiles/en/.

4 Veloci S, Mencarini J, Lagi F et al. Tubercular prosthetic joint infection: two case reports and literature review. Infection 2018; 46: 55-68.

5 Held M, Laubscher M, Dix-Peek S et al. Diagnostic accuracy of the Xpert MTB/RIF assay for extrapulmonary tuberculosis in children with musculoskeletal infections. Pediatr. Infect. Dis. J. 2016; 35: 1165-68.

\title{
Neonatal eosinophilic gastroenteritis mimicking hypertrophic pyloric stenosis
}

\author{
Satoshi Yokoyama, ${ }^{1}$ iD Tatsuo Nakaoka, ${ }^{1}$ Takayuki Nukada, ${ }^{2}$ Yuka Ikeda ${ }^{2}$ and Shigeto Hara ${ }^{2}$ \\ ${ }^{1}$ Departments of Pediatric surgery and ${ }^{2}$ Pediatrics, Japanese Red Cross Society, Wakayama Medical Center, Wakayama, \\ Japan
}

Key words eosinophilic gastroenteritis, food allergy, gastric outlet obstruction, hypertrophic pyloric stenosis, newborn.

Eosinophilic gastroenteritis (EGE) is a disease characterized by eosinophilic infiltration of the gastrointestinal tract. It is known to cause gastric outlet obstruction (GOO) in adults but has been reported rarely in infants presenting with pyloric stenosis, a common form of GOO in children. ${ }^{1}$ We report a neonate presenting with clinical and radiographic findings of pyloric stenosis but with histologic evidence of gastroduodenal EGE on biopsies.

Prenatal ultrasonography had revealed a normal amount of amniotic fluid. The patient was born at 40 weeks 6 days via induced labor because of postdate pregnancy. Birthweight was 2,920 g, and Apgar scores were 9 and 10 at 1 and 5 min after birth. Although she was active and passed meconium after birth,

Correspondence: Satoshi Yokoyama, MD, Department of Pediatric surgery, Japanese Red Cross Society, Wakayama Medical Center, 4-20 Komatsubara-dori, Wakayama, Wakayama, 640-8558, Japan. Email: yokoyama.st@gmail.com

Received 12 April 2019; revised 19 July 2019; accepted 25 September 2019.

doi: $10.1111 /$ ped.14041 she exhibited non-bilious vomiting on day 1. She was being fed with cow's milk-based formula and breast milk. By the second day of life she had lost more than the expected amount of weight and was transferred to the neonatal intensive care unit. Physical examination was unremarkable and peripheral eosinophil count was in the normal range (total white cell count $15500 / \mathrm{mm}^{3}$ with $1.9 \%$ eosinophils). An X-ray demonstrated a dilated stomach, filled with air, with gas in the small intestine and colon. An upper gastrointestinal (UGI) series showed severe GOO and demonstrated a narrowing of the pylorus and little passage, in contrast with the duodenum (Fig. 1a). Ultrasound (US) was performed, demonstrating segmental concentric layered wall thickening in the gastric antrum and pylorus (3 mm thick, $15 \mathrm{~mm}$ in length) and an intact duodenal bulb (Fig. 1b).

A 5 Fr. feeding tube was inserted on Day 3, and she was fed with breast milk and commercial cow's milk formula. At this time, hypertrophic pyloric stenosis (HPS) was suspected but conservative treatment with tube feeding was chosen because of her age. Ten days after the start of tube feeding, improvement in the passage of contrast agent through the 